\title{
Lupus Nephritis or Infections-Whom to Blame?
}

Journal of Postgraduate Medicine, Education and Research (2021): 10.5005/jp-journals-10028-1413

$\begin{array}{ll}\text { CPC Editor } & \text { : Prof Ritambhra Nada } \\ \text { Pathology Discussant } & : \text { Prof Ritambhra Nada }{ }^{1} \\ \text { Clinical Discussant } & : \text { Prof HS Kohli }{ }^{2} \\ \text { Senior Resident } & : \text { Dr Anand Bardia } \\ \text { CPC Chairperson } & : \text { Prof D Behera } \\ \text { DOA } & : 27 / 09 / 2014 \\ \text { DOD } & : 11 / 10 / 2014 \\ \text { Date of CPC } & : 21 \text { January, 2015 }\end{array}$

\section{Case Description}

A 20-year-old woman was admitted with pedal edema, facial puffiness of $5-6$ months duration, along with additional complaints of alopecia, oral ulcers, and photosensitivity. There was no arthralgia/ arthritis or Raynaud's phenomenon. On clinical examination and laboratory investigations, she was found to be anemic ( $\mathrm{Hb}-6.8 \mathrm{mg} /$ $\mathrm{dL}$ ) with pancytopenia (TLC-2,800/ $\mathrm{LL}$, platelet count-119,000/ $\mu \mathrm{L}$ ). She was having nephrotic range proteinuria (24-hour urinary protein-3.2 g/TV) and mild renal dysfunction (S. creatinine-1.3 $\mathrm{mg} / \mathrm{dL}$ ). Her serum complement (C3/C4) was low. Based on the above findings, a diagnosis of systemic lupus erythematosus (SLE) was made with hematological and renal involvement. This was further supported by positive ANA and dsDNA titers. Her pANCA was also positive. Hence, a renal biopsy was performed for histopathological examination which confirmed the presence of crescentic glomerulonephritis due to lupus nephritis (LN) (class IV) along with thrombotic microangiopathy (TMA). She was given MP pulses and oral steroids ( $1 \mathrm{mg} / \mathrm{kg} / \mathrm{day})$. Cell counts improved after steroid treatment. Thereafter, a monthly cyclophosphamide pulse was given according to NIH protocol with oral steroids. Two weeks later, she complained of depressive symptoms. Her renal function deteriorated with rising serum creatinine $(4.8 \mathrm{mg} / \mathrm{dL})$ and blood urea (116). Her platelet count dropped to $78,000 / \mathrm{mL}$ and serum LDH was high (2,200 IU/L). A possibility of TTP/CNS lupus was considered. She was managed with anti-depressants, seven sessions of plasma exchange, and another dose of endoxan in view of worsening of disease activity. Neuropsychiatric symptoms improved and serum creatinine came down to $3.3 \mathrm{mg} / \mathrm{dL}$. However, after a few days, she became febrile with pain and swelling of the left thigh, decreased oral intake, and irritability. A possibility of left thigh cellulitis was considered and managed with piperacillin and tazobactam in emergency OPD. She subsequently became afebrile with a reduction in redness and swelling. Despite this, she remained irritable and had decreased oral intake. Thus, she was readmitted for the third time.

\section{EXAMINATION}

The patient was alert, conscious but irritable. She had pallor with bilateral pedal edema and anasarca. Her pulse rate was normal

\begin{abstract}
1,3 Department of Histopathology, Postgraduate Institute of Medical Education and Research, Chandigarh, India

${ }^{2}$ Departments of Nephrology, Postgraduate Institute of Medical Education and Research, Chandigarh, India

${ }^{4}$ Departments of Pulmonary Medicine, Postgraduate Institute of Medical Education and Research, Chandigarh, India

Corresponding Author: Ritambhra Nada, Department of Histopathology, Postgraduate Institute of Medical Education and Research, Chandigarh, India, Phone: +91 7087008143, e-mail: ritamduseja@yahoo.com

How to cite this article: Nada R, Kohli HS, Bardia A, et al. Lupus Nephritis or Infections-Whom to Blame? J Postgrad Med Edu Res 2021;55(1):53-61.

Source of support: Nil

Conflict of interest: None

(88/minute) with a regular, normal rhythm. Her blood pressure was 120 (systolic)/80 (diastolic) $\mathrm{mm} \mathrm{Hg}$ with a respiratory rate of 22/ minute. She did not have any neck rigidity. In view of her irritable behavior, the sensory examination could not be done. Motor examination revealed a score of $4 / 5$ power on both upper limbs (proximal and distal), $3 / 5$ in the right lower limb (proximal and distal), and 2/5 in the left lower limb (proximal and distal). Deep tendon reflexes could not be done in view of edema. Plantar reflex showed bilateral flexor response. Her left thigh was still swollen with tenderness and ruptured blebs. However, it was relatively better than before.
\end{abstract}

\section{INVESTIGATIONS}

- C3/C4-97/19 mg/dL.

- dsDNA-24.7 IU $/ \mathrm{mL}$ (cut-off 25).

- APLA W/U-b2 glycoprotein-3.25 (<20), anticardiolipin-1.27 $(<20)$, lupus anticoagulant-37.3 (36-50).

- Pus culture and sensitivity - Staphylococcus aureus, sensitive to clindamycin/linezolid and resistant to vancomycin. Subsequent samples were sterile. 
- Blood culture and sensitivity-all three samples were taken at an interval of 2 days were sterile.

- Procalcitonin-3.38 $(<0.5)$.

- Ultrasound of left thigh (24/9/14) - showed marked subcutaneous edema with no obvious collection seen.

- Chest X-ray-shows consolidation in the right mid and lower zone.

- Rest of investigations: refer to Tables 1 to 3.

\section{Course and Management}

This 20-year-old woman was admitted with left thigh cellulitis which showed a partial response to intravenous antibiotics. ${ }^{1,2}$ During her hospital stay for the third time, her symptoms of depression worsened and had decreased verbal output. She also had anemia, thrombocytopenia, hypoalbuminemia, and active sediment in the urine. The antidepressive dose was hiked up. A possibility of disease activity was considered and as there was an apparent resolution of cellulitis, endoxan pulse was repeated in view of neuropsychiatric lupus or active LN, and plasma exchange was started for possible TMA. Subsequently, she also had leukopenia, possibly due to drugs. Cellulitis worsened with the appearance of sloughing. Gastrosurgical consultation was sought and extensive debridement was done under general anesthesia. Multiple blood transfusions (3) were given. Pus and blood culture and sensitivity were repeatedly sterile. Antibiotics were changed to imipenem and vancomycin. G-CSF was administered for leukopenia. There was no improvement in psychiatric symptoms and the patient-reported a high-grade fever. On 8/10/14, she had respiratory distress for which she was intubated and put on intermittent positive pressure ventilation and shifted to the intensive care unit (9/10/14) for further management. Chest X-ray revealed right mid and lower zone consolidation. During ICU stay, she had hypotension, requiring triple ionotropic support to maintain blood pressure. She had a cardiac arrest on 11/10/14 from which she could not be revived.

Table 1: Hemogram findings

\begin{tabular}{lllll}
\hline & $30 / 9 / 14$ & $4 / 10 / 14$ & $7 / 10 / 14$ & $10 / 10 / 14$ \\
\hline $\mathrm{Hb}$ & 7.8 & 9.2 & 6 & 7.8 \\
$\mathrm{TLC} / \mathrm{DLC}$ & 4900 & $\begin{array}{l}2500 / \mathrm{P} 69, \\
\text { L22 }\end{array}$ & 200 & 1500 \\
Platelet & $75 \times 10^{3}$ & $70 \times 10^{3}$ & $21 \times 10^{3}$ & $14 \times 10^{3}$ \\
PBF & $\begin{array}{l}\text { Microcytic/ } \\
\text { hypo }\end{array}$ & $\begin{array}{l}\text { N/No } \\
\text { schitocytes }\end{array}$ & & \\
& hypo & & \\
\hline
\end{tabular}

\section{Case Analysis Final Clinical Diagnosis}

- Systemic lupus erythematosus: class IVLN, TMA, neuropsychiatric manifestations (CNS lupus).

- Left lower limb cellulitis.

- Hospital-acquired pneumonia.

- Septicemia.

\section{Clinical Discussion}

Prof Behera: Thank you Prof Kohli. Please join me here. So, we have just relied on the basic disease of SLE with LN. There have been no comments on differential diagnosis. If there is anyone from the unit to comment upon, please come.

Prof KL Gupta: Good morning. There can be no differential diagnosis in this case. It is a typical case of SLE with LN. The question is why Dr Ritambhra has brought this case to the forum for discussion? This patient had severe SLE with LN and had florid manifestations along with TMA, which is not seen too often. Regarding the terminal events, I guess infection mainly fungal infection could have been the terminal event. Regarding CNS lupus, yes, it is high on cards but it is very difficult to diagnose and manage these cases. Despite the reduction in doses, she was irritable and her condition worsened. At last, the infection took her away.

Prof Behera: Don't you think about vasculitis in this case.

Prof KL Gupta: SLE itself is a secondary cause of vasculitis. The association of SLE with pANCA has been well described in the literature. In fact, we can expect to see some granulomatous change in vessels along with SLE in the autopsy.

Dr Dhooria: Regarding lungs, this patient had low hemoglobin, hypoxemia, and dense consolidation in right lower zone indicating pneumonia. Along with this, she had coagulopathy which could have led to hemorrhages in the lungs. Besides this, she had neutropenia. So, fungal infection is likely. Microangiopathy is also suspected.

Table 3: Arterial blood gas values

\begin{tabular}{lcccc}
\hline \multicolumn{5}{c}{$\begin{array}{l}8 / 10 / 14 \\
(11 \mathrm{pm}):\end{array}$} \\
& $8 / 10 / 14$ & $\mathrm{FiO}_{2}-50 \%$ & $9 / 10 / 14$ & $10 / 10 / 14$ \\
\hline $\mathrm{pH}$ & 7.424 & 7.406 & 7.338 & 7.236 \\
$\mathrm{pO}_{2}$ & 51.7 & 67.1 & 146 & 58.0 \\
$\mathrm{PCO}_{2}$ & 20.9 & 19.8 & 23 & 24.3 \\
$\mathrm{HCO}_{3}$ & 13.4 & 12.2 & 12.2 & 10.1 \\
Saturation & 86.3 & 92.6 & 99 & 82 \\
\hline
\end{tabular}

Table 2: Biochemistry laboratory values and coagulation profile

\begin{tabular}{|c|c|c|c|c|}
\hline $\mathrm{Na} / \mathrm{K} / \mathrm{Cl}$ & $141 / 2.61 / 108$ & $141 / 4.5 / 111$ & & $138 / 3.3 / 112$ \\
\hline Urea/Cr & $213 / 2.39$ & $206 / 2.2$ & $179 / 1.72$ & $176 / 2.0$ \\
\hline OT/PT/ALP & & - & & $26 / 6 / 72$ \\
\hline T Protein/albumin & $3.9 / 1.8$ & $3.8 / 1.7$ & $3.68 / 1.9$ & $3.2 / 1.5$ \\
\hline $\mathrm{Ca} / \mathrm{PO}_{4}$ & $8.7 / 6.3$ & $6.6 / 6.3$ & $7.2 / 5.7$ & $7.0 / 5.7$ \\
\hline LDH & 1099 & - & & - \\
\hline PT/PTI & & $20 / 70 \%$ & $>2$ & $45 / 31 \%$ \\
\hline aPTT/INR & & $32 / 1.46$ & $>2$ & $48 / 3.16$ \\
\hline
\end{tabular}


Prof Behera: I cannot rule out DVT due to infarction in this case. X-ray shows peripheral shadow. I hope the ultrasound of the thigh lesion has probably ruled out DVT and established it as the only cellulitis. Dr Aman, any comments.

Prof Aman: pANCA can be seen in a variety of settings and this can be due to antibodies against various antigens, anti-MPO being one of the most common. So, we should not read too much on pANCA in this case. Regarding vasculitis, this patient had evidence of immune complex-mediated glomerulonephritis, so, one cannot go away from SLE being the cause of vasculitis. As Dr Kohli has already said, seronegative APLA may be the cause of TMA. In addition, this patient had high levels of serum LDH and anemia which supports it. Dr Varun Dhir; There may not be much disease activity in other organs. We can expect chronic changes in the kidney on autopsy. This patient was in immunosuppression and neutropenia in this setting must raise suspicion for any fungal infection like Candida or Aspergillus. The presence of fibrosis, collapsing glomerulopathy (CG) might have led to worsening of renal function.

Prof Manish Rathi: I have two comments to make-(1) The presence of CG may be due to vascular involvement by the disease itself, and (2) Seronegative APLA association has been shown in two to three case series and $<20 \%$ cases, SLE with TMA has been the cause. Out of these, only $5 \%$ had shown the presence of APLA seropositivity. This can be supported by C4d staining as shown in several studies. In this case, seronegative APLA may be the cause for TMA. C4d staining if demonstrated in autopsy might help.

Prof Sanjay Jain: One should look at the positive clinical and laboratory findings. This patient had normal aPTT with no antiphospholipid antibodies except few fibrin thrombi. That should not draw us to diagnosing it as APLA. The more obvious cause is infection leading to sepsis. This is evidenced by the presence of cellulitis, multiple consolidations in lungs, hypoxia, multiple thrombi, and prolonged prothrombin time. It may all due to sepsis. Prof Behera: So, if there are no further comments. To summarize, this patient had SLE with varied manifestations and possibly died due to sepsis at the end. Now may I ask Dr Ritambhra to present the autopsy findings.

\section{Pathology Findings}

A partial autopsy was carried out. The prosecutors noted that the deceased was moderately built. The pleural, peritoneal, and pericardial cavities were within normal limits.

Antenatal kidney biopsy (S-14885/14)—showed the presence of crescentic glomerulonephritis due to LN, class IV.

On partial autopsy, both kidneys weighed $330 \mathrm{~g}$. These were normal in size capsular surface was blotchy. The cut surface also showed a blotchy appearance with distinct corticomedullary distinction. No areas of infarction were noted. Histopathological examination of representative sections showed crescentic glomerulonephritis with diffuse global endocapillary proliferation. In addition, the segmental and global collapse of different ages were also seen. There was diffuse, regenerating tubular necrosis. Arteries showed healed vasculitis (Figs 1 to 4).

Immunofluorescence revealed a full house pattern (IgG, IgM, C3, C1 kappa, and lambda) with extraglomerular staining (Fig. 5). So, a diagnosis of $\mathrm{LN}$, class IV was made.

Lungs-were heavy and together weighed 1,100 g. These were subcrepitant to feel. The pleural surface showed areas of hemorrhagic discoloration. Cut surface revealed patchy areas of hemorrhage, purulent abscesses, and circumscribed necrotic areas with hemorrhagic borders. Histopathological examination from these areas showed hemorrhagic necrosis with microinvasive aspergillosis, Nocardia in purulent abscesses along with Gramnegative pneumonitis. There were areas with cytomegalovirus (CMV) inclusions. There was perifocal edema and hemorrhage (Figs 6 to 8 ).

Liver-weighed 1,200 $\mathrm{m}$ and was grossly normal. Histopathological examination showed areas of centrizonal hemorrhage.

Spleen and lymph nodes-showed lymphodepletion manifested as depletion of white pulp. Germinal centers were atrophic. There was a presence of hemophagocytosis in a few foci.

Colon-showed foci of acute ulceration with the presence of Gram-positive cocci (Fig. 9).

Pancreas-showed obstructive mucous plugs in ducts with mild pancreatitis.

Bone marrow—was hypocellular (Fig. 9).

\section{Final Autopsy Diagnosis (PM-26294)}

In the known cases of SLE with LN.

- Lupus nephritis class IV with collapse.

- Inflammatory myositis.

- Lungs-invasive aspergillosis, nocardiosis, gram-negative pneumonitis with CMV.

- Colon-acute ulceration-gram-positive cocci.

- Lymphodepletion/hemophagocytosis in lymph nodes and spleen.

- Hypocellular bone marrow

- Liver-passive congestion.

- Mild pancreatitis.

\section{Final Discussion}

Prof Behera: Thank you for demonstrating to us the varied manifestations of LN with the presence of CG. The only problem is that autopsy showed multiple polymicrobial infections in the patient which was missed during her lifetime. Now, the protocol is open for discussion.

Prof Kohli: Sir, I would say we "missed the bus" rather than missing the point. Even if we had diagnosed the viral infection during our lifetime, the patient could not survive. The only issue was that she had involvement of the brain by the disease.

Prof KL Gupta: This is an example of LN in its one of the worst forms. It is not common to have LN along with TMA, collapse besides polymicrobial infection including virus, fungus, and bacteria. It is very rare and I wonder if it is seen only in transplant patients or in other cases as well. I have seen four to five cases of SLE with CMV and fungal infection. I did think of infection during the ward rounds in this patient, but we failed to demonstrate any such evidence and then the patient was shifted to the intensive care unit due to worsening condition and finally collapsed. Lupus nephritis as a disease per se is an autoimmune disease and hampers the immunocompetence of the body. Immunosuppressive drugs further add insult to the injury. So, these factors have probably led to the demise of the patient.

Prof Sanjay Jain: It was interesting to see CG. But what about TMA? We anticipated overt TMA as the prothrombin time and other 
coagulation tests were markedly deranged. But pathological examination did not show any thrombosis although there was the presence of subendothelial deposits. So, is it the injury of endothelial cell or surface or the lumen? Were autopsy findings similar to that of the antemortem biopsy which also showed disease activity along with the presence of TMA. It is known that SLE can lead to coagulopathy and immunosuppression. In my viewpoint, initially, it was the disease itself that has done the damage. But at the time of the last admission, it was the infection that led to demise which may be due to the immunosuppressive treatment administered to her for the disease. So, the question arises which is more important to treat first - the disease or the immunosuppression resulting due to the treatment of the disease?

Prof R Nada: Now, it is believed that endothelial cell injury is the root cause of TMA. And it is known that endothelial cells and podocytes crosstalk and sustain the dynamics of renal function. Therefore, any endothelial cell injury in the glomerular capillaries will lead to activation of podocytes and ultimately, a collapse which was seen in this case. So, it is not mandatory to find thrombus for the diagnosis of TMA. Accordingly, there has been a change in terminology to "endotheliosis" to incorporate all these changes which highlight the pathogenesis. Second, antemortem biopsy showed predominantly disease activity while the autopsy findings highlighted the chronic changes. And we have seen from autopsies that podocytes after activation do become dormant over time.
Prof Aman: Disease activity together with infection is a double whammy and it is a challenge to treat these patients. We cannot prevent community-acquired infection but we can take steps to prevent nosocomial infections although it is challenging too.

Prof Manish Rathi: It was unfortunate for the patient to have full house disease activity in the kidneys and full house infection in the lungs. I have two questions. First, I have serious doubts regarding CG. It is because CG in LN is usually associated with an active disease rather than chronic and it is usually seen in minor forms of the disease. Second, if we have sampled the thigh muscles for fungal culture at autopsy.

Prof R Nada: Autopsy for a sampling of thigh muscles requires additional consent, which was not taken in this case. Collapsing glomerulopathy can be seen in both active and inactive LN. In a study by Salvatore et al., CG was observed in 19 patients of LN and all had inactive disease. ${ }^{3}$ In fact, there have been three case reports (two from AlIMS, Delhi), which have reported CG in active LN. Usually, such patients have a bad prognosis. Dr Raja from our institute has a follow-up of patients with CG due to any disease and it was seen that such patients had a worse clinical outcome. Prof Behera: Any additional comments from Prof BD Radotra.

Prof BD Radotra: One should not get swayed by the polymicrobial infections in this case. In my opinion, fungal infection was primarily the dominant cause of septicemia in this patient. One can see the activation of CMV in an immunosuppressive state. An occasional Nocardia abscess cannot lead to such drastic clinical behavior.
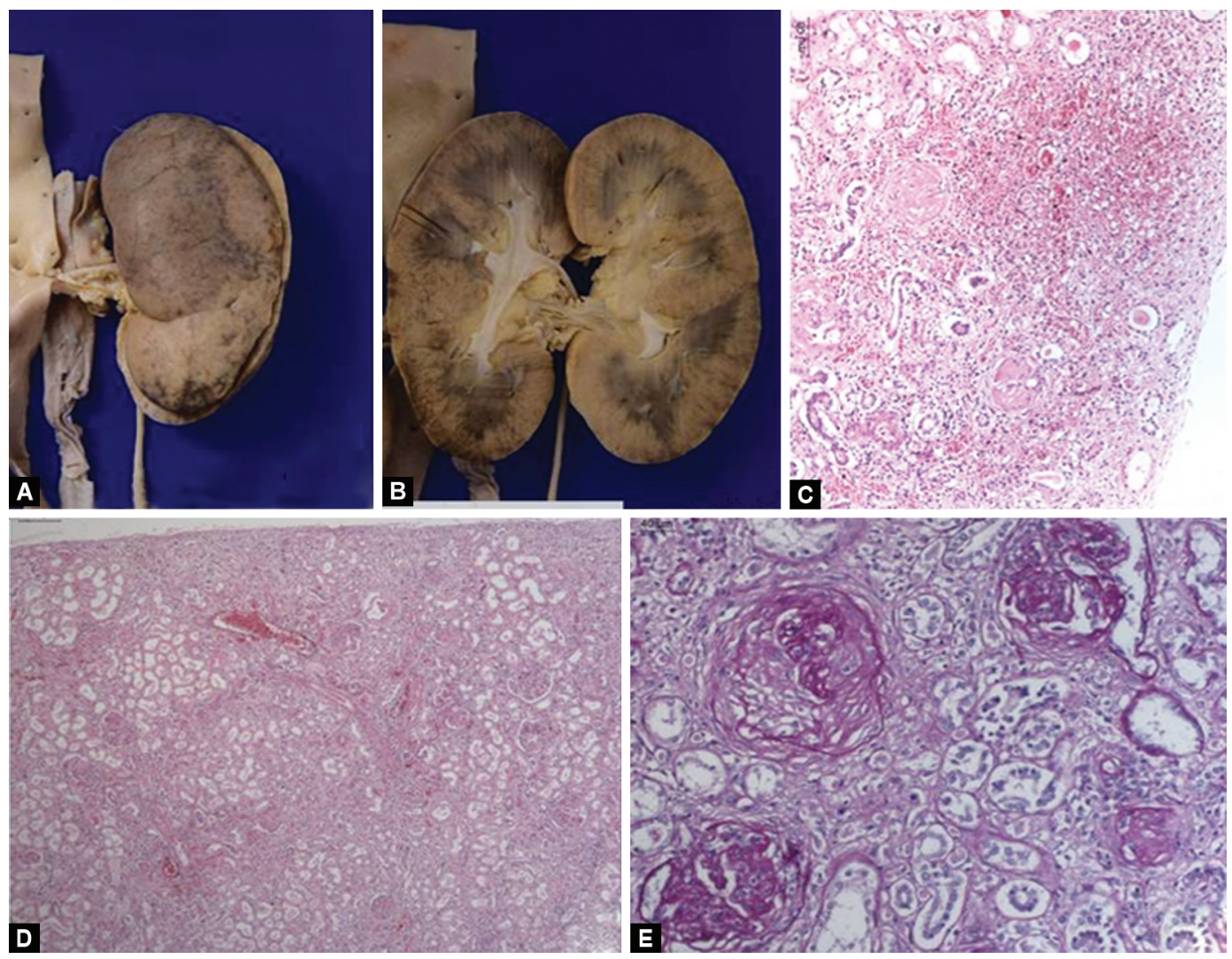

Figs $1 \mathrm{~A}$ to $\mathrm{E}$ : (A and B) Gross photograph of a kidney showing blotchy capsular surface and focal areas of blackish discoloration and distinct corticomedullary junction; (C) Microphotograph from corresponding areas of blackish discoloration show hemorrhage with loss of renal parenchyma (H\&E, 40X); (D) Diffuse involvement of glomeruli presence of crescents (H\&E, 40X); (E) Underlying capillary tuft of glomeruli show diffuse mesangial proliferation with endocapillary hypercellularity fibrocellular crescents (PAS, 200X) 

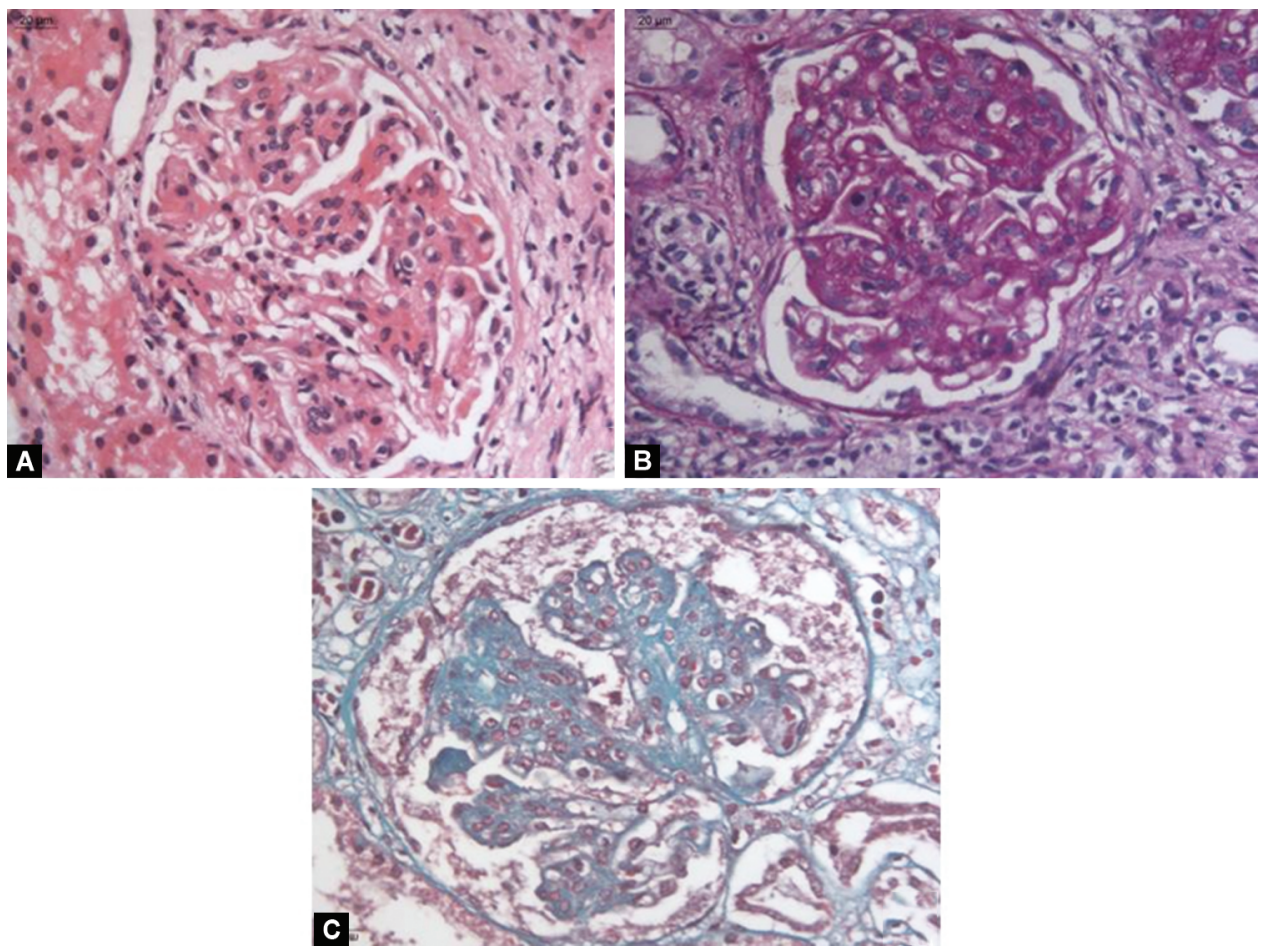

Figs 2A to C: Microphotograph showing endocapillary hypercellularity endothelial cell swelling causing luminal occlusion and thickening of a capillary wall [H\&E (A), PAS (B), and Masson's trichrome stain (C), 400X]
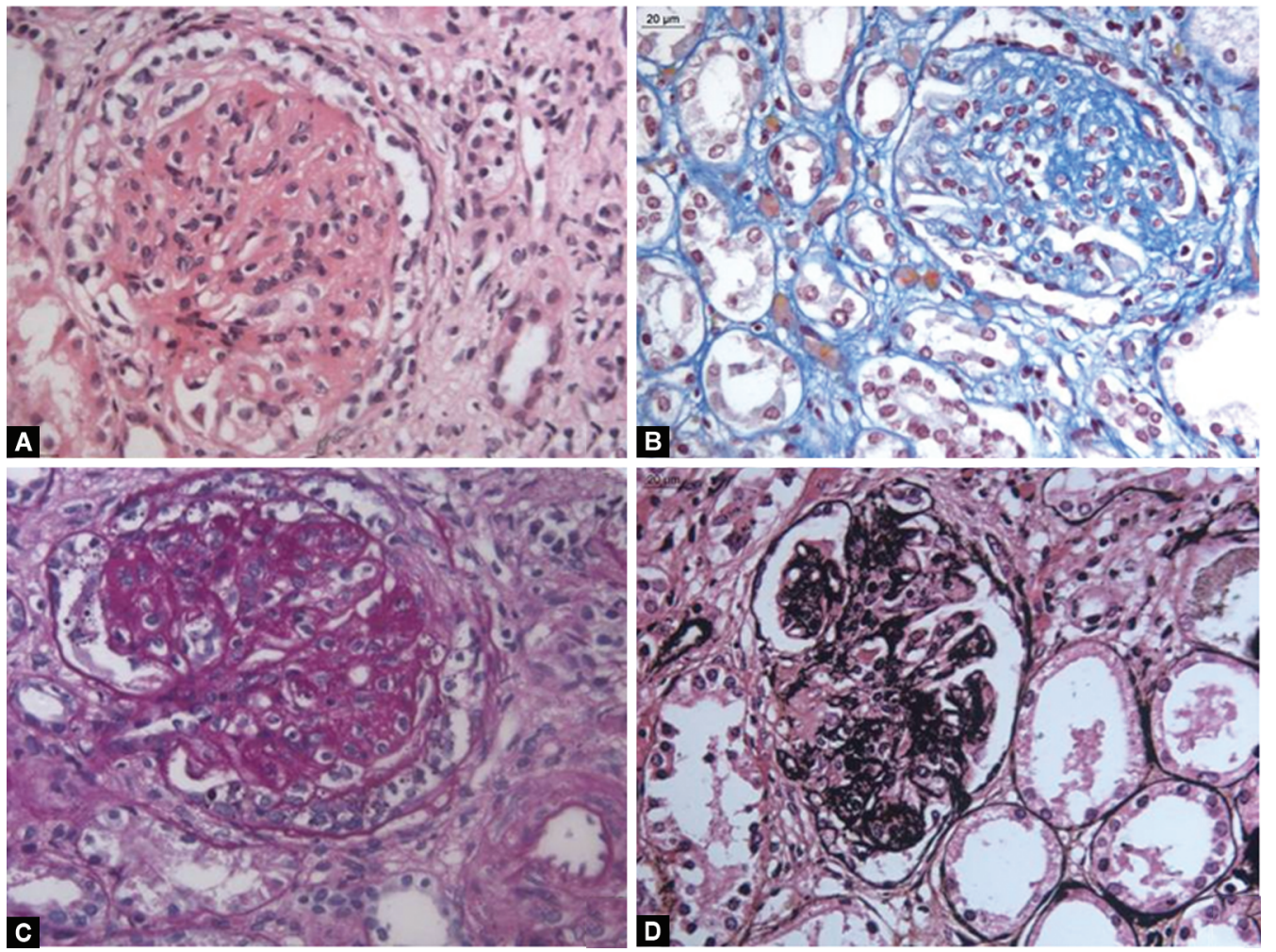

Figs 3 A to D: Microphotograph showing a glomerulus with the collapse of capillary loops and the crowning of podocytes (A to C) and collapsed capillary loops without crowning [H\&E (A), Masson's trichrome stain (B), PAS (C), Jones' silver stain (D), 400×]

Besides, I disagree with Dr Kohli that CNS involvement was probably not there. In view of neurological symptoms and antemortem renal biopsy findings, there must have been vascular damage in
CNS as well. However, we cannot confirm this at present due to partial autopsy.

Prof Behera: Thank you everybody for the participation. 


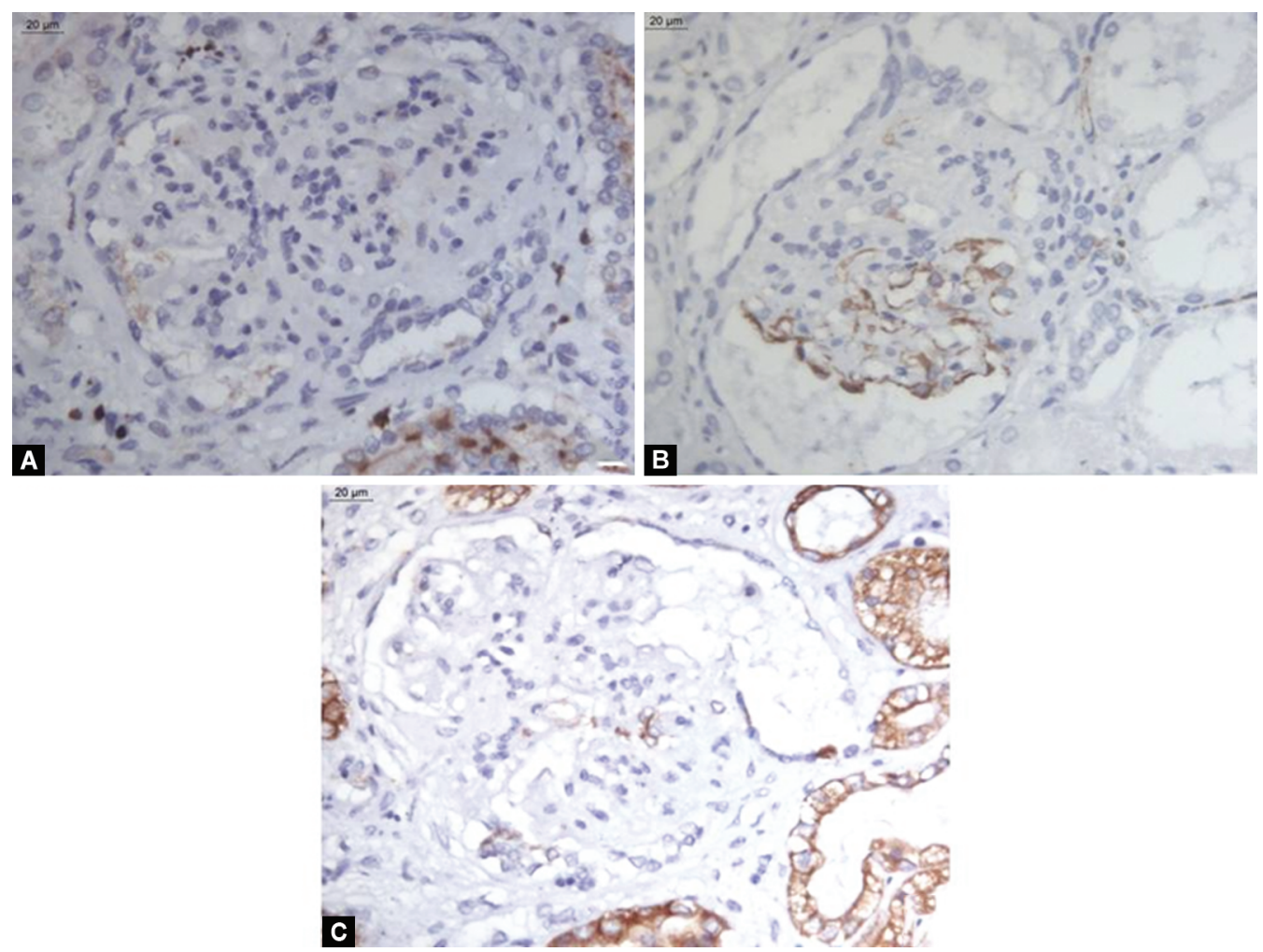

Figs 4A to C: Immunohistochemical stain for Ki67 (A) showing proliferation of podocytes in collapsed glomerular capillary tuft with patchy loss of expression for WT-1 (B) and focal gain cytokeratin 7 (C) [IHC (A to C), 400X]

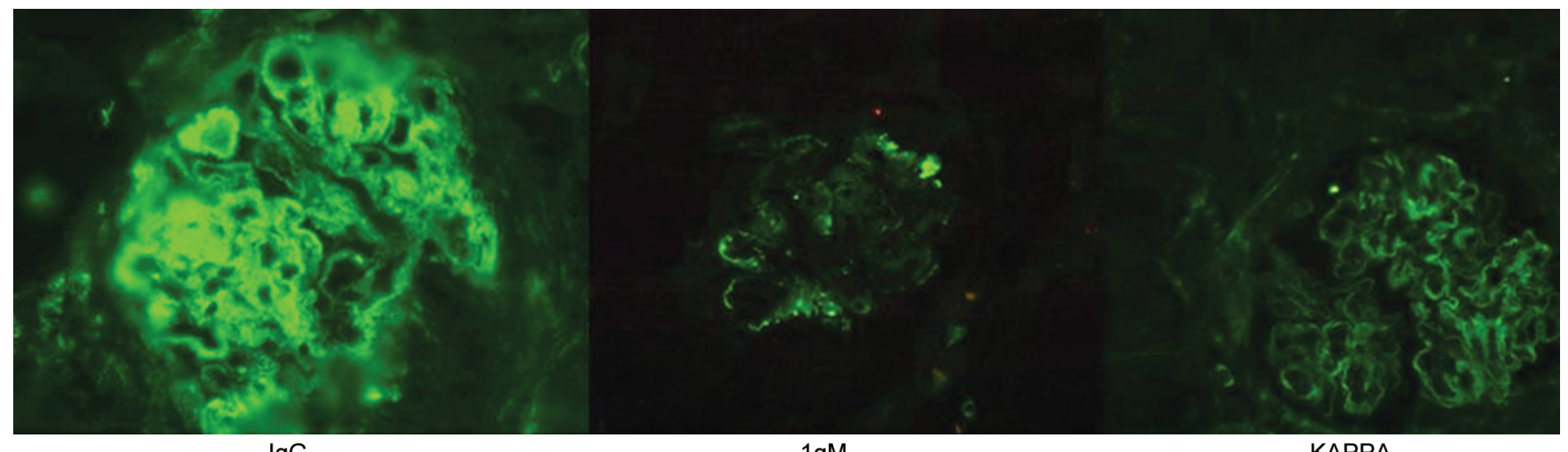

$\lg G$

$1 \mathrm{gM}$

KAPPA

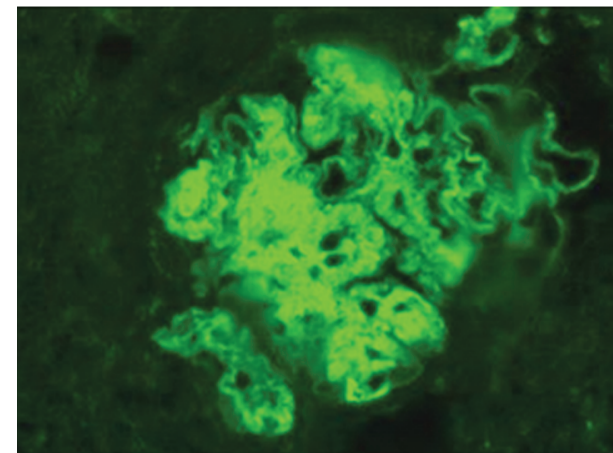

LAMBDA

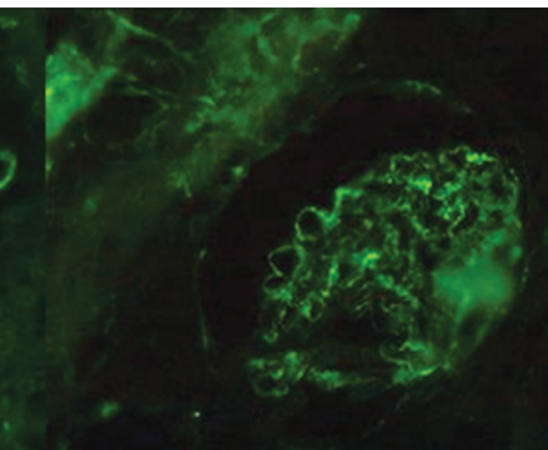

C3

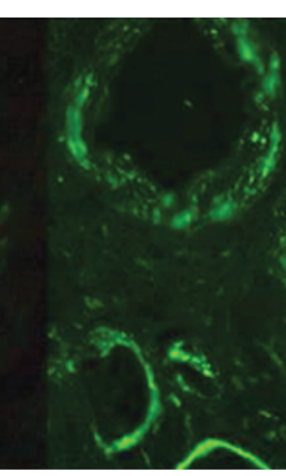

Fig. 5: Direct immunofluorescence showing full house pattern with coarse granular deposits in mesangium and capillary loops for lgG, lgM, kappa, lambda, and C3 along with extraglomerular deposits in blood vessels for lgG (FITC, 400x) 

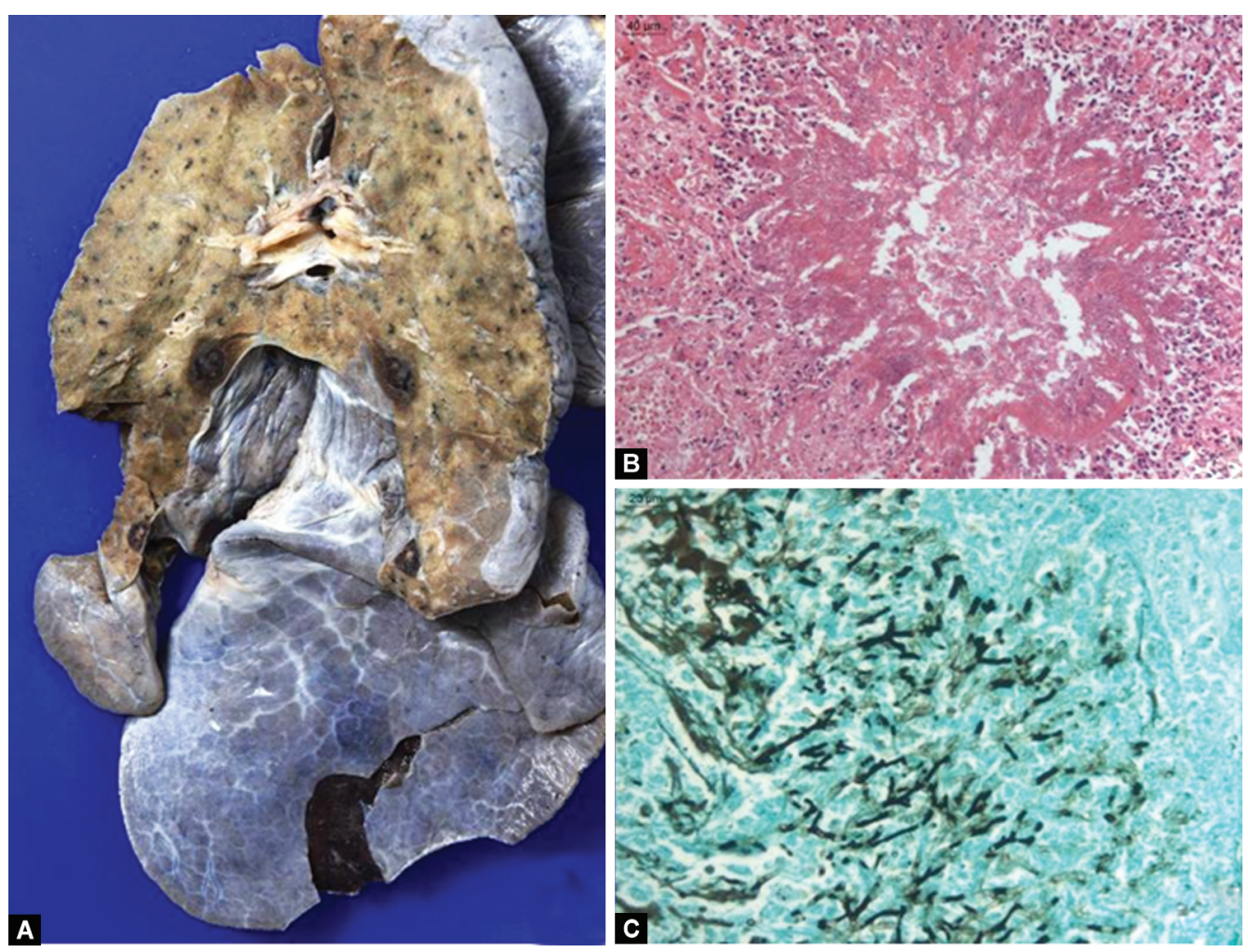

Figs $6 \mathrm{~A}$ to C: Gross photograph of lungs showing hemorrhagic necrotic areas (A), and corresponding microphotograph showing necrosis centered around bronchiole with septate fungal hyphae (B), which are better highlighted on Grocott stain [H\&E (B), Grocott stain (C), (B) 200X (C) 400X]
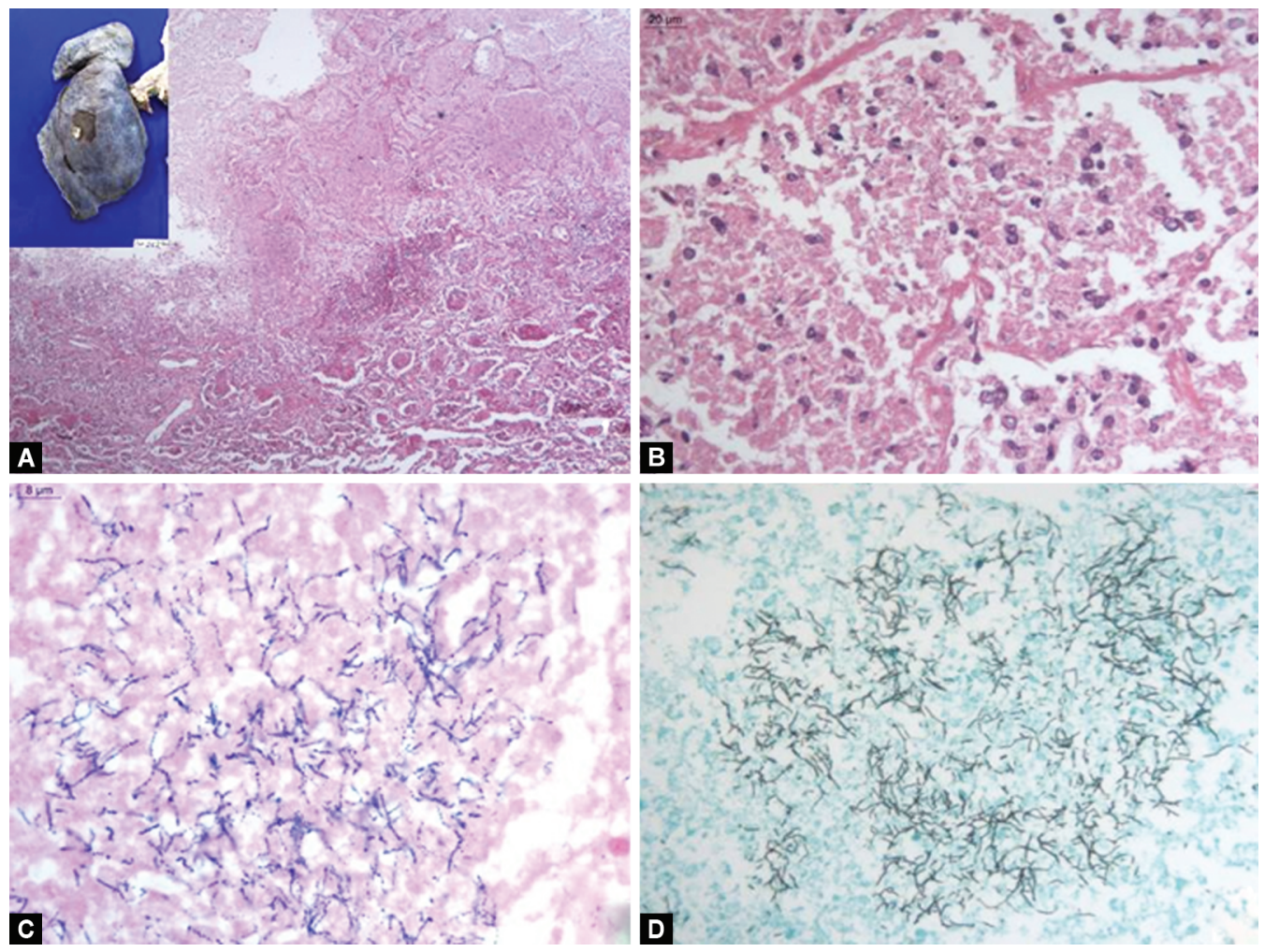

Figs 7A to D: Gross photograph of lungs showing whitish purulent material (inset) and corresponding microphotograph showing acute inflammatory infiltrate with necrosis in H\&E stain. Few filamentous organisms are seen in these areas $[(B), 400 \times]$ which were positive on Gram's stain $[(C), 100 \times]$ and Grocott stain [H\&E (A and B), Gram's stain (C), Grocott stain (D), 40× (A), 100× (B), (C and D) 400×] 

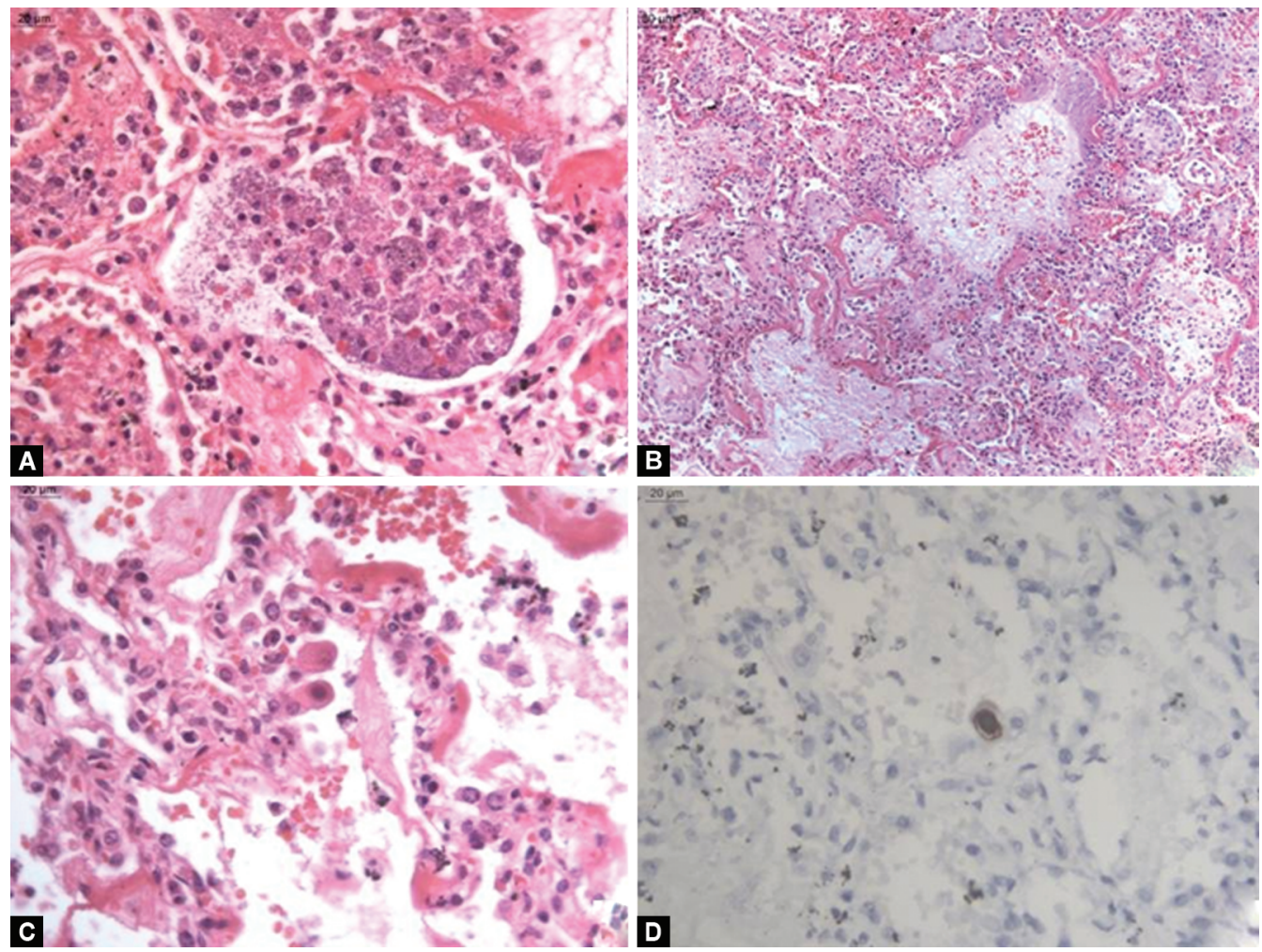

Figs 8A to D: Alveolar spaces were filled with macrophages with granular material which turned out to be gram-negative bacilli on Gram-Twort stain $[(B), 100 \times]$, few endothelial cells had enlarged and smudged nuclei $[(C), H \& E, 400 \times]$ and were confirmed to be cytomegalovirus on immunostain [H\&E (A to C), IHC-CMV (D), 400× (A and B), $100 \times(B$ and D) ]
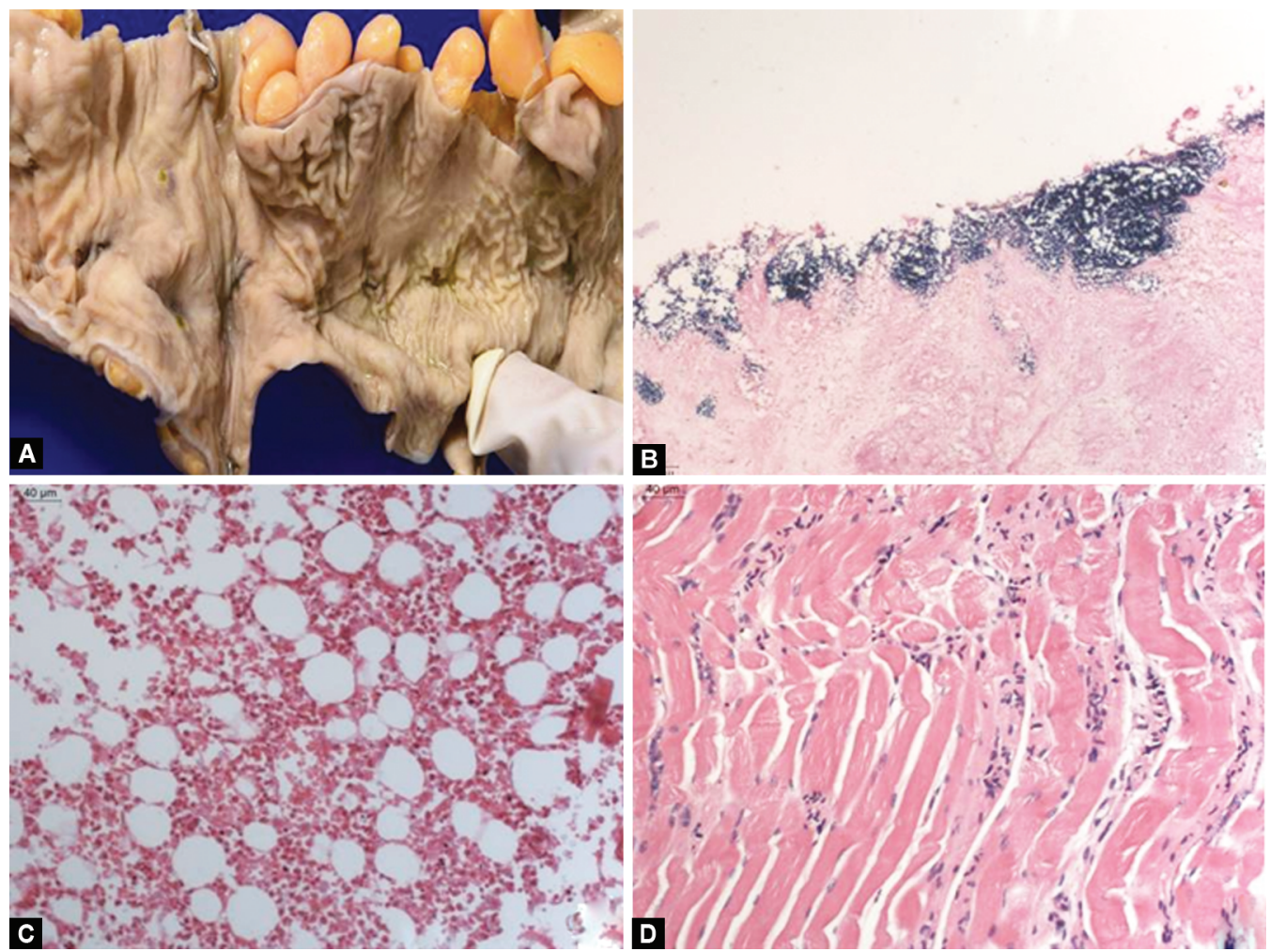

Figs $9 A$ to D: Gross photograph of the colon showing ulceration (A) with corresponding (B) microphotograph showing ulcerated epithelium and colonization by gram-positive bacteria, $(C)$ microphotograph of bone marrow showing hypocellular marrow spaces, (D) microphotograph of psoas muscle with infiltration by lymphomononuclear inflammatory infiltrate [Gram's stain (B), H\&E (C and D), 40× (B), 200× (C and D)] 


\section{Commentary}

The SLE is a multisystemic disease in which there is a production of autoantibodies and immune complexes formation. The antibodies can be directed against many nuclear antigens (ANAs), including DNA, nuclear ARN, and proteins; or against cytoplasmic or cellular membrane antigens. The immune complexes can be deposited or formed in diverse tissues, activating the complement and releasing proinflammatory cytokines that produce tissue damage. The diagnosis of SLE requires a correlation of clinical and laboratory findings. There is renal involvement in the great majority of patients with SLE at some time of the evolution. Renal disease is one of the most frequent causes of death in SLE. Renal manifestations are also very variable, from asymptomatic hematuria or proteinuria until rapidly progressive renal failure and terminal renal damage. Furthermore, LN can be associated with several complications which develop over time and can be part of adverse drug reaction. These include infection, thrombosis, lymphoproliferative disorder, etc.

Thrombotic microangiopathy is not an uncommon vascular change in patients with LN, especially in those with severe diffuse proliferative glomerulonephritis. Thrombotic microangiopathy is found in about 2 and $24 \%$ of patients with LN. ${ }^{4}$ Diagnosis of TMA in SLE is sometimes difficult because these two disorders share similar clinical features, including hemolytic anemia, thrombocytopenia, neurological deficits, renal involvement, and fever. Active SLE disease has been considered as an independent risk factor for the development of TMA, and without careful and extensive studies, TMA may be neglected in SLE patients with high disease activity. The secondary antiphospholipid syndrome should be ruled out. The pathogenesis of TMA in SLE may be multifactorial. This includes low ADAMTS13 levels, SLE disease activity, infection, drugs, etc.

Collapsing glomerulopathy in LN is uncommon and has a bad prognosis. ${ }^{5}$ Collapsing glomerulopathy is a pattern of glomerular injury with a rapid clinical course including massive proteinuria and relative resistance to standard treatment. Multiple etiologies of CG have been described, including certain viral infections (most notably HIV but also parvovirus B19 and hepatitis C), drugs, gene mutations, and vascular occlusion, in addition to idiopathic forms. ${ }^{5}$ Histopathological examination is characterized by the global or segmental collapse of the glomerular capillary tuft, with wrinkling and retraction of the capillary walls overlaid by epithelial cell proliferation in the Bowman space. Collapsing glomerulopathy occurring in the setting of SLE has a strong association with persons of African descent; a typical clinical presentation with heavy proteinuria and often renal insufficiency and frequent progression to ESRD. ${ }^{3}$ The great majority of such patients had the active systemic disease (often a lupus flare) and a significant number had concurrent LN, usually mild (most often International Society of Nephrology/Renal Pathology Society class II). This certainly suggests the involvement of humoral and/ or cell-mediated immunity, both of which have been implicated in the pathogenesis of podocytopathies and SLE. ${ }^{5}$ The relative contributions of various immune pathways may play a key role in determining the responsiveness of these lesions of CG to therapy.

\section{References}

1. Pradhan VD, Badakere SS, Bichile LS, et al. Anti-neutrophil cytoplasmic antibodies (ANCA) in systemic lupus erythematosus: prevalence, clinical associations and correlation with other autoantibodies. J Assoc Physicians India 2004;52:533-537. PMID: 15645975.

2. Fayyaz A, Igoe $A$, Kurien BT, et al. Haematological manifestations of lupus. Lupus Sci Med 2015;2(1):e000078. DOI: 10.1136/lupus-2014000078.

3. Salvatore SP, Barisoni LMC, Herzenberg AM, et al. Collapsing glomerulopathy in 19 patients with systemic lupus erythematosus or lupus-Like disease. Clin J Am Soc Nephrol 2012;7(6):914-925. DOI: 10.2215/CJN.11751111.

4. Chen $\mathrm{M}-\mathrm{H}, \mathrm{Chen} \mathrm{M}-\mathrm{H}, \mathrm{C}$ en $\mathrm{W}-\mathrm{S}$, et al. Thrombotic microangiopathy in systemic lupus erythematosus: a cohort study in North Taiwan. Rheumatology 2011;50(4):768-775. DOI: 10.1093/rheumatology/ keq311.

5. Haas M. Collapsing glomerulopathy in systemic lupus erythematosus: an extreme form of lupus podocytopathy? Clin J Am Soc Nephrol 2012;7(6):878-880. DOI: 10.2215/CJN.03590412. 